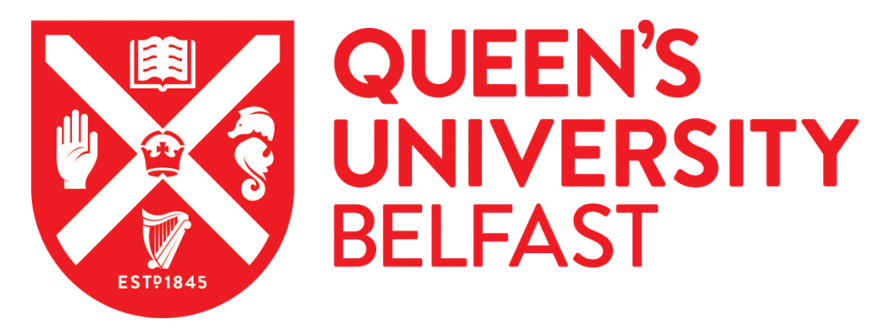

\title{
Oblique electrostatic excitations in a magnetized plasma in the presence of excess superthermal electrons
}

Sultana, S., Kourakis, I., Saini, N., \& Hellberg, M. A. (2010). Oblique electrostatic excitations in a magnetized plasma in the presence of excess superthermal electrons. Physics of Plasmas, 17(3), [032310 ].

https://doi.org/10.1063/1.3322895

Published in:

Physics of Plasmas

Document Version:

Publisher's PDF, also known as Version of record

Queen's University Belfast - Research Portal:

Link to publication record in Queen's University Belfast Research Portal

Publisher rights

(C) 2013 AlP Publishing LLC

\section{General rights}

Copyright for the publications made accessible via the Queen's University Belfast Research Portal is retained by the author(s) and / or other copyright owners and it is a condition of accessing these publications that users recognise and abide by the legal requirements associated with these rights.

Take down policy

The Research Portal is Queen's institutional repository that provides access to Queen's research output. Every effort has been made to ensure that content in the Research Portal does not infringe any person's rights, or applicable UK laws. If you discover content in the Research Portal that you believe breaches copyright or violates any law, please contact openaccess@qub.ac.uk. 


\title{
Oblique electrostatic excitations in a magnetized plasma in the presence of excess superthermal electrons
}

\author{
S. Sultana, ${ }^{1, a)}$ I. Kourakis, ${ }^{1, b)}$ N. S. Saini, ${ }^{1, c)}$ and M. A. Hellberg ${ }^{2, d)}$ \\ ${ }^{1}$ Department of Physics and Astronomy, Centre for Plasma Physics, Queen's University Belfast, \\ BT7 1NN Northern Ireland, United Kingdom \\ ${ }^{2}$ School of Physics, University of KwaZulu-Natal, Private Bag X54001, Durban 4000, South Africa
}

(Received 1 December 2009; accepted 30 December 2009; published online 23 March 2010)

\begin{abstract}
The nonlinear propagation of ion-acoustic waves is considered in a magnetized plasma, composed of kappa distributed electrons and an inertial ion fluid. The fluid-dynamical system of equations governing the dynamics of ion-acoustic waves is reduced to a pseudoenergy-balance equation. The properties of arbitrary amplitude, obliquely propagating ion-acoustic solitary waves are thus investigated via a mechanical-motion analog (Sagdeev potential) approach. The presence of excess superthermal electrons is shown to influence the nature of magnetized ion-acoustic solitons. The influence on the soliton characteristics of relevant physical parameters such as obliqueness (the angle between soliton propagation direction and magnetic field), the electron deviation from a Maxwellian ("superthermality") and the soliton speed is investigated. (C) 2010 American Institute of Physics. [doi:10.1063/1.3322895]
\end{abstract}

\section{INTRODUCTION}

Excess energetic electrons are often present in laboratory, space, and astrophysical plasma environments, viz., the ionosphere, auroral zones, mesosphere, lower thermosphere, etc. ${ }^{1-11}$ Plasmas with an excess of superthermal (nonMaxwellian) electrons are generally characterized by a long tail in the high energy region. The particle distribution therefore deviates significantly from a Maxwellian. ${ }^{1,4,12}$ This type of behavior is often modeled by a kappa $(\kappa)$ type (or generalized Lorentzian) distribution function (df), ${ }^{1,13-15}$ which appears to be more appropriate than a thermal (Maxwellian) distribution in a wide range of plasma situations. The $\kappa$ distribution was first suggested by Vasyliunas ${ }^{1}$ to model space plasmas, and was later adopted by many authors in various physical contexts. A brief review of its physical setting and history can be found in Ref. 16. Noteworthy applications of the $\kappa$-df include, e.g., an interpretation of observations in the Earth's foreshock (for $3<\kappa_{e}<6$ ) ${ }^{17}$ and solar wind models with coronal electrons satisfying $2<\kappa_{e}<6 .{ }^{18,19}$ "Superthermal" plasma behavior was also observed in various experimental plasma contexts, such as laser matter interactions or plasma turbulence. ${ }^{20}$ This seems to be a growing area of study in plasma physics.

It may be pointed out that different forms of the $\kappa$ distribution have appeared in the past, implying different physical scenarios; we refer the reader to the discussion in Ref. 16. Works by Treumann ${ }^{21,22}$ and Collier ${ }^{23}$ have marked the way toward a possible explanation of the apparent ubiquity of kappa distributions, although there is at this stage no comprehensive theory relating this family of distributions to the fundamental underlying physics. However, a very recent

\footnotetext{
a)Electronic mail: ssultana02@qub.ac.uk.

${ }^{b)}$ Electronic mail: i.kourakis@qub.ac.uk.

${ }^{c}$ Electronic mail: ns.saini@qub.ac.uk.

${ }^{\mathrm{d})}$ Electronic mail: hellberg@ukzn.ac.za.
}

study ${ }^{24}$ appears to establish a rigorous link between the $\kappa$ family of distributions and the Tsallis ("q-Gaussian") distribution, ${ }^{25}$ which is intimately linked to the foundations of nonextensive thermodynamics. This association is, however, certainly not algebraically straightforward, and still appears to be a controversial topic.

The analytical expression for the isotropic kappa $(\kappa)$ distribution is given in Refs. 14 and 15, among others. Our analysis in what follows relies on those definitions, so they are not repeated here. The minimum information needed to follow our analysis is provided below. Low values of $\kappa$ represent distributions with a large component of "superthermal particles" (i.e., faster than the thermal speed), which is generally identified as a "hard" spectrum. In what follows we shall use the term "superthermality" as a shorthand description of the properties of such a plasma. At very large values of the spectral index $\kappa$, the velocity df approaches a Maxwellian distribution. It should therefore be noted that earlier results (for thermalized plasma particles) are readily recovered in the algebra, in the infinite $\kappa$ limit. One requires $\kappa>3 / 2$ for physical reasons, since the effective thermal velocity is defined as $\theta=[(\kappa-3 / 2) / \kappa]^{1 / 2}\left(2 k_{B} T / m\right)^{1 / 2}$; here $T$ is the kinetic temperature and $m$ the mass of a given particle species. ${ }^{16}$ The deviation of the kappa family of distributions from the Maxwell-Boltzmann distribution is reflected in the analytical expression for the species number density, ${ }^{15}$ a fact which affects the charge balance and yields consequences which will be made obvious in our analysis below.

A comprehensive nonlinear theory for arbitrary amplitude ion-acoustic (IA) solitary waves was suggested by Sagdeev and co-workers ${ }^{26,27}$ a few decades ago. That method relied on a mechanical-motion analog, associating solitary waves in unmagnetized plasmas with a fictitious problem of a point mass motion in a pseudopotential (often referred to as the Sagdeev potential). Other work using this approach has considered various plasma compositions beyond the standard 
electron-ion $(e-i)$ model,${ }^{28-31}$ and also, for instance, a superthermal background. ${ }^{32}$ This last paper, ${ }^{32}$ in particular, explored the effect of excess superthermal particles (through the parameter $\kappa$ ) on the behavior and characteristics of IA solitons in an unmagnetized plasma. The Sagdeev theory was later extended to cover oblique pulse propagation with respect to an ambient magnetic field, ${ }^{33-37}$ but that required the assumption of quasineutrality.

The scope of our investigation is to analyze the effect of electron superthermality, modeled by a kappa distribution, on the oblique propagation characteristics of nonlinear IA excitations in an electron-ion plasma in the presence of a uniform external magnetic field. We shall use the pseudopotential method to investigate the occurrence and characteristics of oblique arbitrary amplitude IA waves. The range of permitted Mach number values for the existence of solitary IA waves in a magnetized kappa plasma will be determined, and in particular we will demonstrate their dependence on superthermality (via $\kappa$ ). We shall also investigate the effect of obliqueness on the Mach number and on the soliton characteristics. A comparison to the case of a thermal (Maxwellian) plasma will be carried out at all stages.

Importantly, recent results suggest that the pseudopotential formalism may fail to describe large-amplitude structures propagating at large angles to the field; in particular, the stronger the obliquity the lower the amplitude has to be, in respect for Maxwell's laws. "Weak" (finite, yet not too significant) obliqueness is therefore implicitly assumed everywhere below.

This paper is organized as follows. In Sec. II, we present the governing model equations. In Sec. III, the characteristics of linear waves are briefly summarized, focusing on the influence of $\kappa$ and obliqueness in comparison to earlier results. In Sec. IV, we explain the method for arbitrary amplitude solitary wave theory and derive a Sagdeev type pseudopotential. We determine the permitted velocity range for solitary structures in Sec. V. A parametric investigation in terms of various relevant parameters follows in Sec. VI. Finally, we summarize our results in the concluding Sec. VII.

\section{GOVERNING MODEL EQUATIONS}

We consider a two-component collisionless plasma embedded in a uniform magnetic field, composed of the following:

(1) cold ions (charge $q_{i}=+Z e$, mass $m_{i}$ ), described by the fluid-moment equations and

(2) electrons $\left(q_{e}=-e\right.$, mass $\left.m_{e}\right)$, assumed to obey an isotropic kappa velocity distribution.

\section{A. The model}

We take the ambient magnetic field $\mathbf{B}_{0}=B_{0} \hat{z}$ to lie along the $z$-axis (unit vector $\hat{z}$ ). To simplify the analysis, we assume that propagation occurs in the $x-z$ plane (hence setting $\partial / \partial y=0$ below); see Fig. 1.

The fluid continuity and momentum equation(s) for the ions, ignoring thermal pressure effects, read

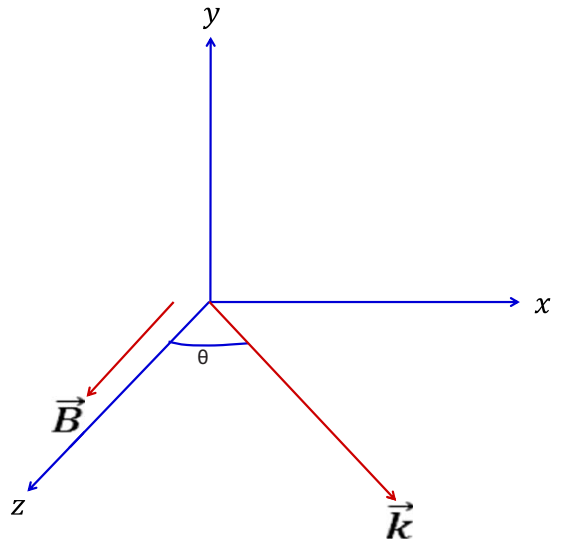

FIG. 1. (Color online) All fluid variables depend on a traveling stationary frame, where $\vec{B}=(0,0, B), \vec{k}=\left(k_{x}, 0, k_{z}\right)$, and the direction cosines $\alpha=k_{x} / k$ $=\sin \theta$ and $\beta=k_{z} / k=\cos \theta$.

$$
\begin{aligned}
& \frac{\partial n_{i}}{\partial t}+\nabla \cdot\left(n_{i} \mathbf{u}_{i}\right)=0, \\
& \frac{\partial \mathbf{u}_{i}}{\partial t}+\left(\mathbf{u}_{i} \cdot \nabla\right) \mathbf{u}_{i}=-\frac{q_{i}}{m_{i}} \nabla \Phi+\frac{q_{i}}{m_{i}}\left(\mathbf{u}_{i} \times \mathbf{B}_{0} \hat{z}\right),
\end{aligned}
$$

where $n_{i}, u_{i}$, and $\Phi$ are the ion number density, the ion mean velocity, and the electrostatic potential, respectively.

We adopt a kappa distribution for the electrons ${ }^{15}$

$$
n_{e}=n_{e 0}\left[1-\frac{e \Phi}{\left(\kappa-\frac{3}{2}\right) k_{B} T_{e}}\right]^{-\kappa+1 / 2} \text {. }
$$

The real parameter $\kappa$ measures the deviation from the Maxwell-Boltzmann equilibrium (which is recovered in the limit $\kappa \rightarrow \infty$ ). The rigorous way to close the system of evolution equations, for electrostatic perturbations, would be to couple the species through Poisson's Equation

$$
\nabla^{2} \Phi=-4 \pi e\left(Z n_{i}-n_{e}\right) .
$$

Charge neutrality at equilibrium implies $n_{i 0} Z-n_{e 0}=0$, where the index " 0 " denotes the unperturbed (equilibrium) number density values.

Instead of using Eq. (4), we shall, for analytical tractability in the nonlinear algebraic manipulation to follow, adopt the plasma approximation or charge neutrality hypothesis, namely, $Z n_{i} \simeq n_{e}$, i.e., assuming that the right-hand side (rhs) of Eq. (4) vanishes. This implies the physical assumption that spatial variation in the electric potential is slow, essentially occurring on a scale far beyond the Debye sphere. $^{27}$

\section{B. Scaling}

The normalized ion continuity and momentum equations take the form of a system of (scalar) equations,

$$
\begin{aligned}
& \frac{\partial n}{\partial t}+\frac{\partial\left(n u_{x}\right)}{\partial x}+\frac{\partial\left(n u_{z}\right)}{\partial z}=0, \\
& \frac{\partial u_{x}}{\partial t}+\left(u_{x} \frac{\partial}{\partial x}+u_{z} \frac{\partial}{\partial z}\right) u_{x}=-\frac{\partial \phi}{\partial x}+\frac{\Omega_{i}}{\omega_{p i}} u_{y},
\end{aligned}
$$




$$
\begin{aligned}
& \frac{\partial u_{y}}{\partial t}+\left(u_{x} \frac{\partial}{\partial x}+u_{z} \frac{\partial}{\partial z}\right) u_{y}=-\frac{\Omega_{i}}{\omega_{p i}} u_{x}, \\
& \frac{\partial u_{z}}{\partial t}+\left(u_{x} \frac{\partial}{\partial x}+u_{z} \frac{\partial}{\partial z}\right) u_{z}=-\frac{\partial \phi}{\partial z},
\end{aligned}
$$

where $u_{i}, n_{i}$, and $\Phi$ are scaled as follows: $u=u_{i} / c_{s}, n$ $=n_{i} / n_{i 0}$, and $\phi=\Phi / \Phi_{0}$, respectively. Here, $n_{i 0}$ is the equilibrium ion density and $\Omega_{i}$ the ion-cyclotron frequency. Space and time variables are scaled by the Debye length $\lambda_{D e}$ $=\left(k_{B} T_{e} / 4 \pi n_{e 0} e^{2}\right)^{1 / 2}=\left(k_{B} T_{e} / 4 \pi Z n_{i 0} e^{2}\right)^{1 / 2}$, and the inverse ion plasma frequency $\omega_{p i}^{-1}=\left(4 \pi n_{i 0} Z^{2} e^{2} / m_{i}\right)^{-1 / 2}$, respectively. Finally, the potential scale reads $\Phi_{0}=k_{B} T_{e} / e$. The characteristic speed scale used for velocity normalization is then $c_{s} \equiv\left(Z k_{B} T_{e} / m_{i}\right)^{1 / 2}$.

The normalized form of Poisson's equation [Eq. (4)] is

$$
\frac{\partial^{2} \phi}{\partial x^{2}}+\frac{\partial^{2} \phi}{\partial z^{2}}=\left(1-\frac{\phi}{\kappa-\frac{3}{2}}\right)^{-\kappa+1 / 2}-n \text {. }
$$

As indicated above, we shall normally adopt here the plasma approximation which consists in considering a vanishing rhs in Eq. (9), viz.,

$$
n \simeq\left(1-\frac{\phi}{\kappa-\frac{3}{2}}\right)^{-\kappa+1 / 2}
$$

Although this physically reduces our scope to perturbations on a length-scale greater than the order of magnitude of the Debye length, ${ }^{27}$ this choice is imposed by analytical tractability constraints, once obliqueness in the propagation direction is considered. We note, in passing, that the scaled form of our analytical model is independent of the value of the ion charge state $Z$. This would no longer be true if a third species were present in the background $[Z$ would then appear in the charge balance via the rhs of Eq. (9)].

We shall now proceed by considering the system of Eqs. (5)-(8) and (10) (i.e., using the plasma approximation) in the nonlinear analysis in Sec. IV and beyond. However, as a special case we shall first use Poisson's equation [Eq. (9)] rather than the quasineutrality assumption (10) when dealing with the linear dynamics in Sec. III.

\section{LINEAR DISPERSION CHARACTERISTICS}

The linear dispersion relation (DR) for wave propagation can be obtained from the linearized Eqs. (5)-(9). After a short algebraic manipulation, this may be cast in the form

$\omega_{ \pm}^{2}=\frac{1}{2}\left(r+\frac{k^{2}}{\nu+k^{2}}\right) \pm \frac{1}{2}\left[\left(r+\frac{k^{2}}{\nu+k^{2}}\right)^{2}-4 r \frac{k_{z}^{2}}{\nu+k^{2}}\right]^{1 / 2}$,

where $\nu=(\kappa-1 / 2) /(\kappa-3 / 2), r=\left(\Omega_{i} / \omega_{p i}\right)^{2}$, and $k_{z}=k \cos \theta$. Note the explicit dependence of Eq. (11) on the following:

(i) the electron superthermality via $\nu$ (Maxwellian behavior is recovered for $\nu \rightarrow 1$, i.e., $\kappa \rightarrow \infty)$,

(ii) the magnetic field via the frequency ratio $r$, and

(iii) the propagation direction (obliqueness) via $k_{z}$ and, hence, $\theta$.

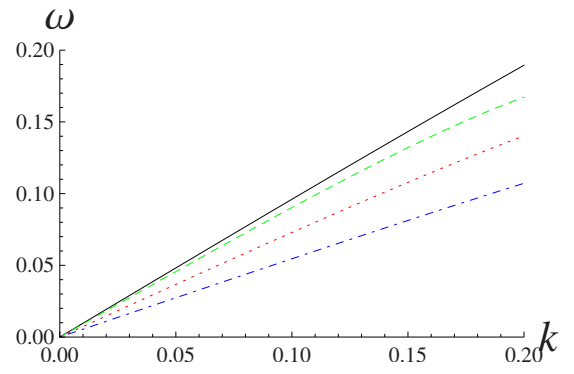

FIG. 2. (Color online) Examples of the linear DR of obliquely propagating IA waves. From top to bottom: Solid curve for $\kappa=15$ and $r=0$; dashed curve for $\kappa=15, \cos \theta=0.95$, and $r=0.05$; dotted curve for $\kappa=3, \cos \theta=0.95$, and $r=0.05$; and dot-dashed curve for $\kappa=2, \cos \theta=0.95$, and $r=0.05$.

Two distinct dispersion curves are obtained, for the plus/minus sign. The lower curve $\omega_{-}$represents obliquely propagating magnetized IA waves. We note that $\omega_{-}=0$ at $k=0$, and for $k \ll 1, \omega_{-} \approx k \cos \theta / \sqrt{\nu}$. Furthermore, one can show that the phase speed $v_{\mathrm{ph}}^{(-)}$falls off for larger $k$, thus suggesting that it possesses an upper boundary at $v_{\mathrm{ph}, \max }^{(-)}$ $=\cos \theta / \sqrt{\nu}$ for small $k$.

The upper curve $\omega_{+}$represents obliquely propagating ion-cyclotron waves, as one can easily see from the fact that $\omega_{+} \rightarrow r$ (in normalized units) as $k \rightarrow 0$, i.e., the frequency tends to the ion-cyclotron frequency. For $k \ll 1$, this mode behaves as $\omega_{+}^{2} \approx r+\left(\sin ^{2} \theta / \nu\right) k^{2}$ from which one sees that $v_{\mathrm{ph}}^{(+)} \rightarrow \infty$ as $k \rightarrow 0$. We also note that at $k=0$ there is a frequency gap between $\omega_{+}$and $\omega_{-}$equal to $r$.

In the absence of a magnetic field $(r \rightarrow 0)$ Eq. (11) becomes

$$
\omega_{+}^{2}=\frac{k^{2}}{\nu+k^{2}},
$$

which is the usual DR for unmagnetized IA waves. The same result is obtained for parallel propagation (setting $\theta \rightarrow 0$ ). Interestingly, the phase speed of this acoustic mode near zero is reduced by a factor $1 / \sqrt{\nu}$ as compared with its equivalent in Maxwellian plasmas, i.e., $\lim _{k \rightarrow 0}\left(\omega_{+} / k\right)=1 / \sqrt{\nu} \leq 1$.

As expected, earlier results on linear waves in superthermal plasmas are recovered from the above expressions. The $\kappa$-dependent plasma dispersion function was investigated in full detail from first principles in Refs. 39 and 40 (and recently revisited in Ref. 41). As an application of the theory, electrostatic modes were investigated in those papers, in particular focusing on Langmuir ${ }^{39}$ and obliquely propagating IA modes. ${ }^{40}$ We note here that, upon restoring dimensions in Eq. (11), one recovers exactly the DR, which appears as Eq. (68) in Ref. 40 [noting that our $\kappa$-dependent screening factor $\nu$ defined above is incorporated in the definition of $\lambda_{\kappa, e}$ appearing in (69) therein]; cf. Fig. 1(a) therein.

Figure 2 depicts the linear DR obtained for the acoustic mode $\omega_{-}$from Eq. (11), for a weakly magnetized plasma $(r=0.05)$. The solid curve corresponds to $\omega_{+}$in the quasiMaxwellian limit (large $\kappa=15$ ) for $r=0$, and is provided for reference. As expected, the ion-cyclotron wave behaves like an IA wave for $r=0$. The wave frequency (and phase velocity) decreases with an increasing superthermal component 


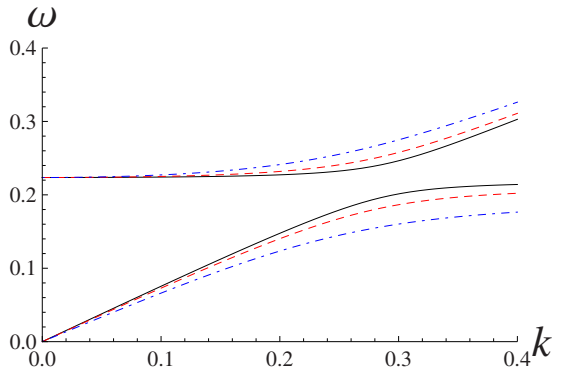

FIG. 3. (Color online) The linear DR for electrostatic waves propagating obliquely in a magnetized plasma for $\kappa=3$ and $r=0.05$. Upper (three) curves: $\omega_{+}$and lower (three) curves $\omega_{-}$. Solid curves for $\cos \theta=0.98$; dashed for $\cos \theta=0.95$; and dot-dashed for $\cos \theta=0.87$.

(i.e., $\kappa$ decreasing) for fixed obliqueness and magnetization. The same effect (lower phase speed) is found for increased obliqueness (i.e., reducing $\cos \theta$ ), and for stronger magnetization.

In Fig. 3, the upper three curves represent the ioncyclotron mode, while the lower three are for the IA branch. We see that the phase speed of the IA wave decreases with increasing obliqueness, while the effect on the ion-cyclotron wave is the reverse, namely, higher phase speed for increased obliqueness, thus leading to an increased overall separation between the two modes. Finally, the effect of excess superthermality (stronger for lower $\kappa$ ) is depicted in Fig. 4. We note that an increase in the superthermal electron component causes the phase speed of both the ion-cyclotron and IA waves to decrease. In both of the latter Figures, the frequency cutoff at $k=0$, for the upper mode, is left intact, since it depends only on the strength of the magnetic field.

As pointed out earlier, we shall be using the quasineutrality assumption when dealing with the nonlinear behavior and hence we turn next to the DR obtained from the system of Eqs. (5)-(8) if closed by Eq. (10), namely,

$$
\omega_{ \pm}^{2}=\frac{1}{2}\left(r+\frac{k^{2}}{\nu}\right) \pm \frac{1}{2}\left[\left(r+\frac{k^{2}}{\nu}\right)^{2}-4 r \frac{k_{z}^{2}}{\nu}\right]^{1 / 2} \text {. }
$$

We note the disappearance of the screening term present in Eq. (11); specifically, the latter equation leads to Eq. (13) upon formally substituting $k^{2} /\left(\nu+k^{2}\right) \rightarrow k^{2} / \nu$. The DR given in Eq. (13) differs from that obtained in Refs. 34 and 36 only due to the presence of superthermal electrons (viz., those are

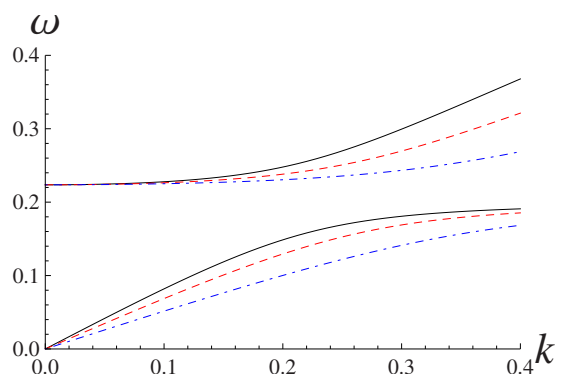

FIG. 4. (Color online) The linear DR for electrostatic waves propagating obliquely in a magnetized plasma for $\cos \theta=0.95$ and $r=0.05$. Upper (three) curves: $\omega_{+}$and lower (three) curves: $\omega_{-}$. Solid curves for $\kappa=8$; dashed for $\kappa=3$; and dot-dashed for $\kappa=2$. recovered for $\nu \rightarrow 1$ ). In particular, setting $\nu \rightarrow 1$ in Eq. (13) one recovers exactly Eq. (12) in Ref. 34, which also matches Eq. (11) in Ref. 36 (ignoring thermal pressure therein).

Exploring the limiting behavior of the DR found in Eq. (13), one can easily show that for $k \rightarrow 0$, the wave behavior is the same as found above. In particular, the phase velocity of the lower (acoustic) mode has a maximum value for $k \rightarrow 0$, viz., $v_{\mathrm{ph}}^{(-)}=\cos \theta / \sqrt{\nu}$ for $k \rightarrow 0$, and decreases with $k$. On the other hand, for small $k$ the phase velocity of the upper (cyclotron) mode is infinite, decreasing with $k$, to a minimum value for the opposite limit, $k \rightarrow \infty$, where it turns out that $\omega_{+} \approx k \cos \theta / \sqrt{\nu}$, yielding a minimum phase speed, $v_{\mathrm{ph}}^{(+)} \approx \sqrt{1 / \nu}$. For $\kappa \rightarrow \infty$, viz., $\nu \rightarrow 1$, these remarks agree with the results in Ref. 34 .

We note therefore that there is a region of phase speeds, $\left[v_{\mathrm{ph}, \text { max }}^{(-)}, v_{\mathrm{ph}, \min }^{(+)}\right]$in which linear modes do not propagate, i.e., in the interval $\left[\nu^{-1 / 2} \cos \theta, \nu^{-1 / 2}\right]$. We shall later see that, not surprisingly, this is exactly the velocity region in which nonlinear excitations are allowed to occur.

Finally, we may add that our analysis of oblique linear modes appears to be related to the Gould-Trivelpiece modes, studied (for Maxwellian plasmas) in the 1960s. ${ }^{42}$ Interestingly, when $\theta=0$, the two linear modes have the same phase velocity, and the range of velocities available for nonlinear excitations tends to zero. It is easy to show that in fact the two modes then reduce to the standard IA wave (with unnormalized $\omega=k c_{S}$ ) and an upper mode with un-normalized $\omega=\Omega_{i}$. Clearly the latter mode is spurious as the magnetic field cannot affect a parallel propagating longitudinal wave. ${ }^{42}$

\section{ARBITRARY AMPLITUDE SOLITARY WAVE THEORY}

Anticipating the existence of arbitrary amplitude traveling solitary waves, we shall assume that all fluid variables in the evolution equations depend on a single traveling variable $\xi=\alpha x+\beta z-M t$ (where $M$ is the normalized pulse propagation velocity, and $\alpha, \beta$ are the direction cosines along the $x$ and $z$ directions, respectively; thus, $\alpha^{2}+\beta^{2}=1$ ) shown in Fig. 1. It must be added for rigor, that although the normalizing speed $c_{s}$ is the expression for the sound speed in an electron-ion plasma with a Boltzmann electron distribution, it does not correspond to the true sound speed in our case, which is $\kappa$-dependent. Indeed, Debye shielding is altered in a plasma with a $\kappa$ distribution, and thus an effective $\kappa$-dependent Debye length is found, as pointed out in Refs. 43-46. Therefore, $M$ is not truly the Mach number in our case.

The above traveling wave ansatz generally leads to a set of coupled ordinary differential equations in the moving coordinate $\xi$; details can be found, e.g., in Refs. 29 and 30. Applying the coordinate transformation to the system of Eqs. (5)-(8), the fluid equations become

$$
\begin{aligned}
& -M \frac{d n}{d \xi}+\alpha \frac{d\left(n u_{x}\right)}{d \xi}+\beta \frac{d\left(n u_{z}\right)}{d \xi}=0 \\
& \left(-M+\alpha u_{x}+\beta u_{z}\right) \frac{d u_{x}}{d \xi}+\alpha \frac{d \phi}{d \xi}-\frac{\Omega_{i}}{\omega_{p i}} u_{y}=0,
\end{aligned}
$$




$$
\begin{aligned}
& \left(-M+\alpha u_{x}+\beta u_{z}\right) \frac{d u_{y}}{d \xi}+\frac{\Omega_{i}}{\omega_{p i}} u_{x}=0, \\
& \left(-M+\alpha u_{x}+\beta u_{z}\right) \frac{d u_{z}}{d \xi}+\beta \frac{d \phi}{d \xi}=0 .
\end{aligned}
$$

We may now integrate Eqs. (14)-(17) by applying appropriate boundary conditions for localized perturbations, viz., $n \rightarrow 1, u_{x, z} \rightarrow 0$, and $\phi \rightarrow 0$ at $\xi \rightarrow \pm \infty$. We thus obtain

$$
\begin{aligned}
& n\left(\alpha u_{x}+\beta u_{z}\right)=M(n-1), \\
& u_{z}=\frac{\beta}{M}\left(\int n d \phi-1\right), \\
& u_{x}=\frac{M}{\alpha}\left(1-\frac{1}{n}\right)-\frac{\beta^{2}}{M \alpha}\left(\int n d \phi-1\right),
\end{aligned}
$$

where we have taken quasineutrality, i.e., Eq. (10) into account. A tedious yet straightforward algebraic manipulation leads to

$$
\frac{d^{2} S}{d \xi^{2}} \equiv \frac{d^{2}}{d \xi^{2}}\left[\phi+\frac{M^{2}}{2}\left(1-\frac{\phi}{\left(\kappa-\frac{3}{2}\right)}\right)^{2 \kappa-1}\right]=F(\phi),
$$

where $S$ obviously denotes the quantity within square brackets and

$$
\begin{aligned}
F(\phi)= & \frac{\Omega_{i}^{2}}{\omega_{p i}^{2}}\left[\left(1+\frac{\beta^{2}}{M^{2}}\right)\left(1-\frac{\phi}{\left(\kappa-\frac{3}{2}\right)}\right)^{-\kappa+1 / 2}\right. \\
& \left.-1-\frac{\beta^{2}}{M^{2}}\left(1-\frac{\phi}{\left(\kappa-\frac{3}{2}\right)}\right)^{-2 \kappa+2}\right] .
\end{aligned}
$$

Multiplying both sides of Eq. (21) by $d S / d \xi$ and integrating once, we obtain

$$
\frac{1}{2}\left(\frac{d \phi}{d \xi}\right)^{2}+\Psi(\phi ; M, \kappa, \beta)=0,
$$

where the (Sagdeev-type) pseudopotential $\Psi(\phi ; M, \kappa, \beta)$ is given by

$$
\begin{aligned}
\Psi(\phi ; & M, \kappa, \beta) \\
= & \frac{\Omega_{i}^{2}}{\omega_{p i}^{2}}\left[1-\frac{M^{2}\left(\kappa-\frac{1}{2}\right)}{\left(\kappa-\frac{3}{2}\right)}\left(1-\frac{\phi}{\left(\kappa-\frac{3}{2}\right)}\right)^{2 \kappa-2}\right]^{-2} \\
& \times\left[1+\frac{\beta^{2}}{2 M^{2}}+\beta^{2}+\frac{M^{2}}{2}-\left(M^{2}+\beta^{2}\right)\left(1-\frac{\phi}{\left(\kappa-\frac{3}{2}\right)}\right)^{\kappa-1 / 2}\right. \\
& +\left(1-\frac{\beta^{2}\left(\kappa-\frac{1}{2}\right)}{\left(\kappa-\frac{3}{2}\right)}\right) \phi-\left(1+\frac{\beta^{2}}{M^{2}}\right)\left(1-\frac{\phi}{\left(\kappa-\frac{3}{2}\right)}\right)^{-\kappa+3 / 2} \\
& \left.+\frac{\beta^{2}}{2 M^{2}}\left(1-\frac{\phi}{\left(\kappa-\frac{3}{2}\right)}\right)^{-2 \kappa+3}+\frac{M^{2}}{2}\left(1-\frac{\phi}{\left(\kappa-\frac{3}{2}\right)}\right)^{2 \kappa-1}\right] .
\end{aligned}
$$

The detailed derivation of the latter three relations can be found in the Appendix.

Equation (23) can be regarded as the analog of an energy-balance equation in mechanics for the mechanical problem of motion of a unit mass located at "position" $\phi$ and evolving in "time" $\xi$, at a "speed" $d \phi / d \xi$, in a potential $\Psi(\phi ; M, \kappa, \beta)$. We recall, in view of the forthcoming analysis that the Maxwellian limit ${ }^{34}$ is recovered for $\kappa \rightarrow \infty$. So, our findings (for finite $\kappa$ ) are to be compared to the results in Ref. 34.

In order for solitary solutions to exist, ${ }^{26,30}$ the following requirements must be fulfilled:

$\Psi(\phi=0)=d \Psi(\phi) /\left.d \phi\right|_{\phi=0}=0 \quad$ and $\quad d^{2} \Psi(\phi) /\left.d \phi^{2}\right|_{\phi=0}$ $<0$, so that the origin is a maximum of the function $\Psi$. Also, as imposed by the reality of $\phi$, from Eq. (23),

(ii) $\Psi(\phi)$ must be negative in the region $0<\phi<\phi_{\max }$; here $\phi_{\max }$ denotes a positive root of $\Psi$, for positive potential excitations to exist (a similar relation should hold on the negative semiaxis, for negative structures to occur). The nature of these requirements is clarified in the figures provided below.

\section{SOLITON SPEED RANGE}

The origin at $\phi=0$ defines the equilibrium state, which should represent a local maximum of the pseudopotential $\Psi(\phi)$. From Eq. (24), it is clear that $\Psi(\phi=0)=d \Psi(\phi=0) /$ $d \phi=0$ is satisfied. By imposing the convexity requirement of the second derivative being negative at the origin, one defines a region of velocity values where solitary excitations may exist. This is therefore often referred to as the soliton existence condition. ${ }^{30}$ In our case, we thus obtain the condition

$$
\left.\frac{d^{2} \Psi}{d \phi^{2}}\right|_{\phi=0}=\frac{\Omega_{i}^{2}}{\omega_{p i}^{2}} \frac{M^{2}-M_{1}^{2}}{\left(M^{2}-M_{2}^{2}\right) M^{2}}<0,
$$

where

$$
M_{1}=|\beta|\left(\frac{2 \kappa-3}{2 \kappa-1}\right)^{1 / 2} \leq 1
$$

and

$$
M_{2}=\left(\frac{2 \kappa-3}{2 \kappa-1}\right)^{1 / 2}=\nu^{-1 / 2} .
$$

Equation (25) can also be written in the form

$$
\left.\frac{d^{2} \Psi}{d \phi^{2}}\right|_{\phi=0}=\frac{\Omega_{i}^{2}}{\omega_{p i}^{2}}\left(\frac{\beta^{2}}{M^{2}}+\frac{1-\beta^{2}}{M^{2}-M_{2}^{2}}\right)<0 .
$$

We draw the conclusion, from Eq. (25) [or Eq. (28)] that for a fixed value of $\kappa$, soliton solutions may exist only for values of the Mach number in the interval

$$
M_{1}<|M|<M_{2},
$$

i.e.,

$$
\cos \theta<\frac{|M|}{M_{2}}<1,
$$

which is the region of permitted $M$ values for a given value of the angle $\theta$ between the propagation direction and the magnetic field. We shall henceforth omit the absolute value, 
since positive velocity $M$ values will be assumed everywhere below. Recall that $\beta=\cos \theta \leq 1$, since it is defined as a direction cosine.

A few comments are in order, regarding the physical interpretation of the above soliton existence criterion. First of all, it is straightforward to see that the $\kappa$-dependent expression (27) for $M_{2}=\nu^{-1 / 2}$ is the true sound speed in an unmagnetized two-component plasma with kappa-distributed electrons, normalized with respect to the conventional IA speed, $c_{s} \equiv\left(Z k_{B} T_{e} / m_{i}\right)^{1 / 2}$. Hence the expression in Eq. (30), $M / M_{2}$, represents the Mach number of the soliton relative to the true unmagnetized sound speed.

One normally expects solitons to exist only for "superacoustic speeds." Thus it appears at first sight to be extremely surprising to find, as we may see from Eq. (29) that $M_{2}$ is the upper limit for soliton existence, and not the lower limit. However, in this context, it is important to recognize that $M_{2}$ is not the phase speed of the magnetized IA wave, which, as we have seen in Sec. III, is given (in our normalization) by $\cos \theta / \sqrt{\nu}$ in the ultrashort-wavelength limit, i.e., by $M_{1}$. In the Maxwellian limit, $\nu \rightarrow 1$, and the lower Mach number limit becomes $\cos \theta$. The correction factor $\nu^{-1 / 2}$ in the case of a kappa distribution was seen earlier to be the factor by which the Debye length, say $\lambda_{D}^{(\kappa)}$ (and hence the unmagnetized sound speed $\left.\omega_{p, i} \lambda_{D}^{(\kappa)}\right)$, decreases due to excess superthermality of the electrons [note the screening term $\nu$ in the denominator of Eqs. (11) and (12) above]; recall that this quantity reduces to unity for infinite $\kappa$. An important aspect is that this lower Mach number limit for soliton existence varies with the obliqueness through $\cos \theta$. We therefore conclude that these obliquely propagating magnetized IA solitary waves are indeed supersonic, a fact that appears to have escaped the attention of the authors of Ref. 34.

On the other hand, $M_{2}$ is the minimum phase speed of the upper mode (cyclotron wave) in the magnetized model, as we have seen in Sec. III. This acts as the upper limit of accessible soliton speeds. Again, we note that for the Maxwellian case $(\kappa \rightarrow \infty ; \nu \rightarrow 1)$, it follows that $M_{2} \rightarrow 1$.

In the light of the above, one overcomes one's first surprise at finding that $M<M_{2}$ everywhere, a fact which appears to contradict the known result for supersonic parallel propagation. ${ }^{26,32}$ In fact, it was pointed out and briefly discussed years ago for Maxwellian plasmas in Ref. 34 that the region $[\cos \theta<M<1]$ was determined to be the existence condition for obliquely propagating solitons in $e-i$ plasmas with Maxwellian electrons, where, as here, $M$ was normalized with respect to the standard IA speed in a Maxwellian $e-i$ plasma. As we have seen, this result is indeed readily recovered from our Eq. (29) above for infinite $\kappa$.

One observes, therefore that in a plasma with kappa distributed electrons, the range of permitted Mach number values $\left[M_{1}, M_{2}\right]=\left[\nu^{-1 / 2} \cos \theta, \nu^{-1 / 2}\right]$ for obliquely propagating IA solitons coincides with the range of values which is forbidden for linear wave phase speeds; cf. the discussion in Sec. III above. Clearly, when $\cos \theta \rightarrow 1$ (parallel propagation), the model, which is based on propagation at an angle to a magnetic field, breaks down, as the range of accessible Mach numbers tends to zero.

The variation in $M_{1}$ and $M_{2}$ (solid line in Fig. 5) with $\kappa$

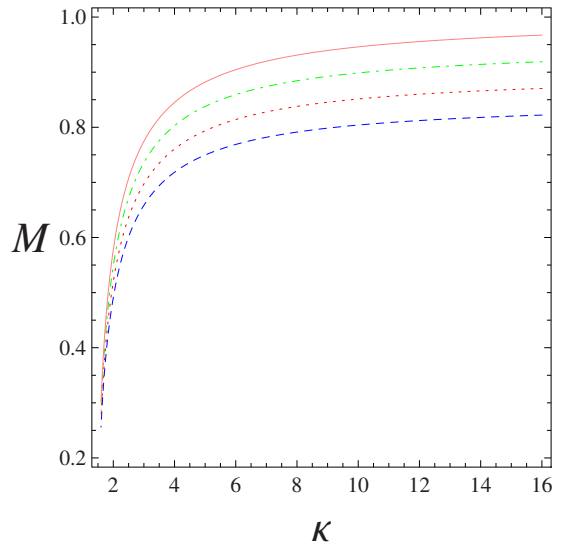

FIG. 5. (Color online) The velocity threshold for the existence of solitary structures is obtained by imposing the condition $\Psi^{\prime \prime}(\phi=0)<0$. The variation in the critical Mach number $M_{1}$ with $\kappa$. From top to bottom: Solid curve: $\beta=1$ [corresponding to $M_{2}$ in Eq. (28)]; dot-dashed curve: $\beta=0.95$; dotted curve: $\beta=0.9$; and dashed curve: $\beta=0.85$.

(for fixed $\beta$ ) and also with $\beta$ (for fixed $\kappa$ ) are shown in Figs. 5 and 6 , respectively. We see that $M_{1}$ is reduced as propagation goes more oblique, thus extending the region of permitted $M$ values as the angle $\theta$ between magnetic field and propagation direction is increased. We may add for completeness, that an extra constraint on the values of $M$ could be expected to come from the requirement that the density should be real, thus $\phi$ must not exceed some maximum value, say $\phi_{m}=\kappa-3 / 2$ [see Eq. (10)]. We have checked, by solving the equation $\Psi(\kappa-3 / 2, M, \kappa, \beta)=0$ that $\Psi\left(\phi_{m}\right)$ is always positive (thus the limit is never reached; remember that $\Psi(\phi)<0$ is imposed physically), so this constraint is not of relevance here.

Concluding, the positive potential obliquely propagating solitary wave solutions of the ion fluid system of equations exist for values of the Mach number $M$ in the range $M \in\left[M_{1}, M_{2}\right]=\left[\nu^{-1 / 2} \cos \theta, \nu^{-1 / 2}\right]$. Recalling the definition $\nu^{-1 / 2}=[(2 \kappa-3) /(2 \kappa-1)]^{1 / 2}$, we see that both upper and lower limits vary with $\kappa$, while only the lower limit varies with obliqueness.

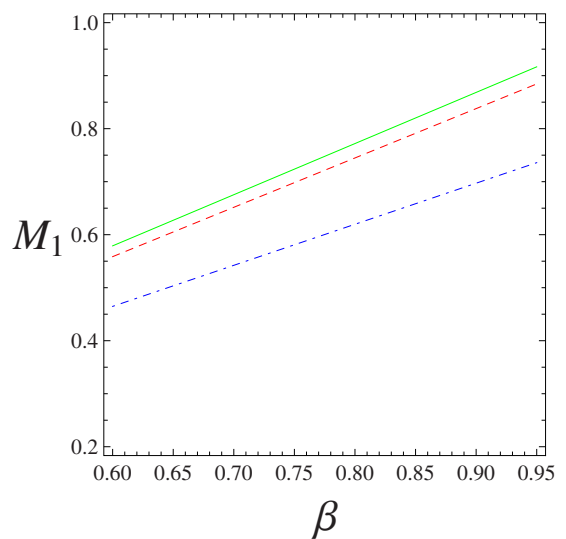

FIG. 6. (Color online) Variation in the Mach number threshold, $M_{1}$ with $\beta$. From top to bottom: Solid curve: $\kappa=15$; dashed curve: $\kappa=8$; and dot-dashed curve: $\kappa=3$. 


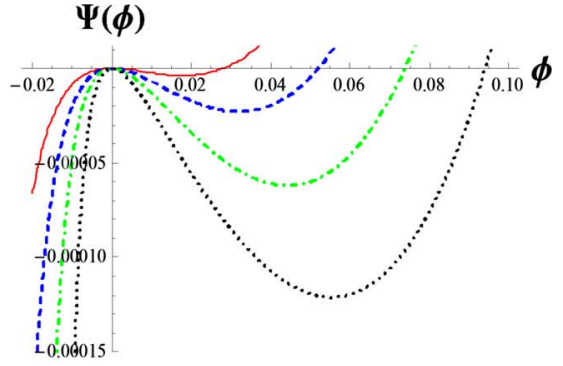

(a)

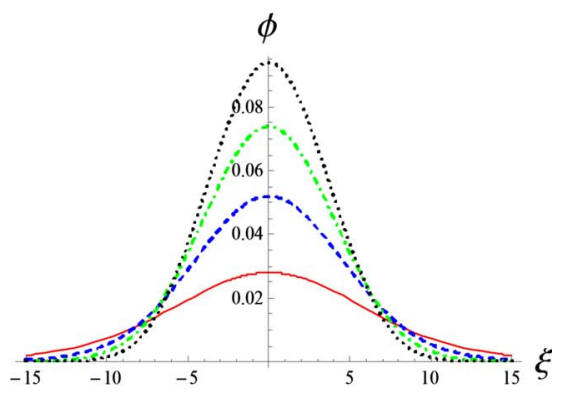

(b)

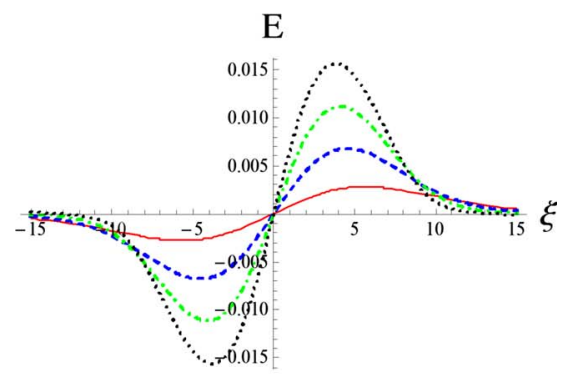

(c)

FIG. 7. (Color online) (a) Variation in the pseudopotential $\Psi(\phi)$ with $\phi$ for $\kappa=2, \beta=0.9, \Omega_{i}=0.5, \omega_{p i}=1.4$, and different values of Mach number, $M$. From top to bottom: Solid curve: $M=0.53$; dashed curve: $M=0.54$; dotdashed curve: $M=0.55$; dotted curve: $M=0.56$. (b) The corresponding electrostatic potential perturbations and (c) the resulting electric field, obtained numerically.

\section{PARAMETRIC INVESTIGATION}

We recall that the pseudopotential function $\Psi(\phi ; M, \kappa, \beta)$ depends on the electric potential $\phi$ and thus also has a parametric dependence on the relevant physical parameters: the excitation speed $M$, the electron nonthermality (via the parameter $\kappa$ ), and the obliqueness of propagation (via $\beta=\cos \theta$ ). We shall now investigate the effect of each of these parameters separately. We need to remember that the form of the pseudopotential $\Psi$, in fact, predicts the shape of the potential pulse: the value of its root (where the curve crosses the axis) corresponds to the pulse amplitude, while the depth of the pseudopotential, as expressed by Eq. (24), is associated with the maximum value of the slope of potential curve $\phi(\xi)$.

\section{A. Velocity dependence}

By plotting the pseudopotential $\Psi$ for fixed values of plasma parameters, we see in Fig. 7 that the amplitude increases as $M$ is increased. It is also found that the depth of

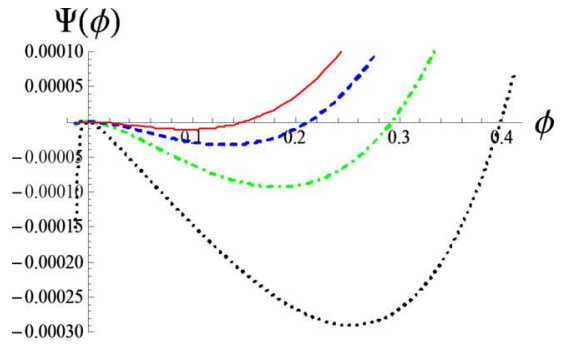

(a)

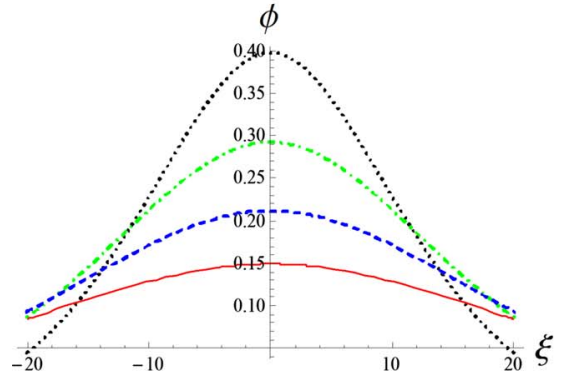

(b)

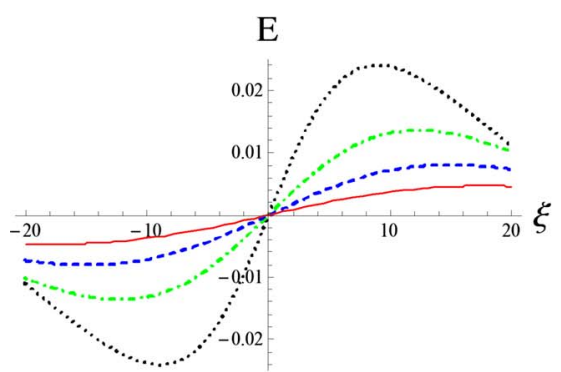

(c)

FIG. 8. (Color online) (a) Increased superthermality effect (via $\kappa$ ) for $\beta=0.8, M=0.8, \Omega_{i}=0.2$, and $\omega_{p i}=1.3$. From top to bottom: Solid curve: $\kappa=10$; dashed curve: $\kappa=7$; dot-dashed curve: $\kappa=5$; dotted curve: $\kappa=3.5$. (b) The corresponding electrostatic potential perturbations and (c) the resulting electric field, obtained numerically.

the Sagdeev potential well (and hence the steepness of the soliton profile) increases as the Mach number is increased within the range $M_{1}<M<M_{2}$. This is in agreement with the earlier result of Ref. 32 for unmagnetized plasma, which also holds for parallel propagation.

\section{B. Superthermality effect (via $\kappa$ )}

The effect of an increase in superthermal electrons on linear IA waves propagating in a magnetized plasma was studied in Ref. 40. It may be noted that Landau damping of electrostatic plasma waves is often enhanced in the presence of a superthermal electron population, as compared with Maxwellian plasmas. ${ }^{47}$

In the framework of our model, we have investigated the superthermality effect for a fixed soliton propagation speed $(M)$ within the accessible range. It is found that the soliton amplitude increases with decreasing $\kappa$, which agrees with the results of Ref. 32. Also, the soliton profile becomes steeper for a greater excess of superthermal electrons; see Fig. 8. 


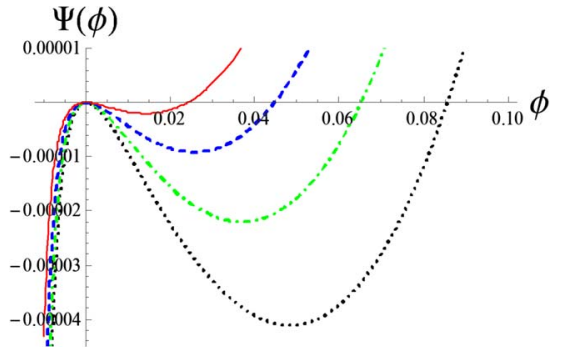

(a)

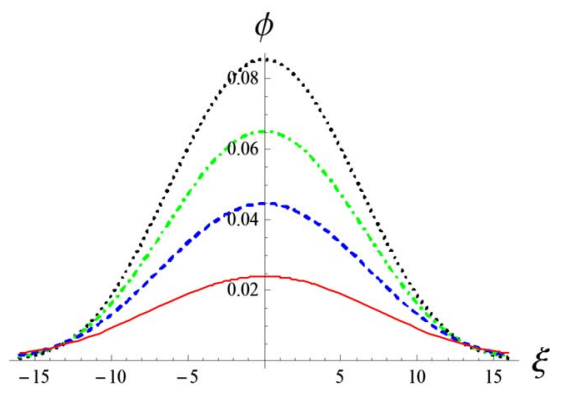

(b)

FIG. 9. (Color online) (a) Effect of obliqueness (via $\beta$ ) on the pseudopotential $\Psi(\phi)$ for $\kappa=3, M=0.76, \Omega_{i}=0.5$, and $\omega_{p i}=1.5$. From top to bottom: Solid curve: $\beta=0.97$; dashed curve: $\beta=0.96$; dot-dashed curve: $\beta=0.95$; dotted curve: $\beta=0.94$. (b) The corresponding electrostatic potential perturbations.

This behavior arises due to the fact that the threshold Mach number decreases in the presence of more superthermal electrons (decreasing $\kappa$ ); hence a given $M$ is increasingly greater than the lower limit, and thus larger amplitude solitary waves are generated. These considerations are in full agreement with Ref. 32 .

\section{Obliqueness effect}

The effect of the obliqueness of the propagation direction, as expressed via $\beta(=\cos \theta)$, has been analyzed for highly superthermal electrons, taking $\kappa=3$, and also for a large (quasi-Maxwellian) value $\kappa=15$. In both cases, as the obliqueness, i.e., the angle $(\theta)$ between the directions of wave propagation and the external magnetic field, $B_{0}$, increases, the amplitude of the solitary waves increases. This can be interpreted via a simple argument, in fact borrowing insight from earlier knowledge for the unmagnetized case $^{32}$ and hence for parallel propagation. As the obliqueness increases (hence $\cos \theta$ decreases), the critical Mach number threshold $M_{1}$ decreases (see Fig. 6). Therefore, it follows that a given $M$ value exceeds the lower critical value by a larger amount, and thus the amplitude of the solitary structure increases.

It is seen from Figs. 9 and 10 that a more nearly parallel situation (smaller propagation angle) leads to smaller excitations. It should be pointed out, for rigor that for large angles the assumptions that the waves are electrostatic is no longer valid, and we should look for fully electromagnetic structures. This was emphasized in recent criticism of oblique models for arbitrary amplitude excitations; ${ }^{38}$ in fact, the

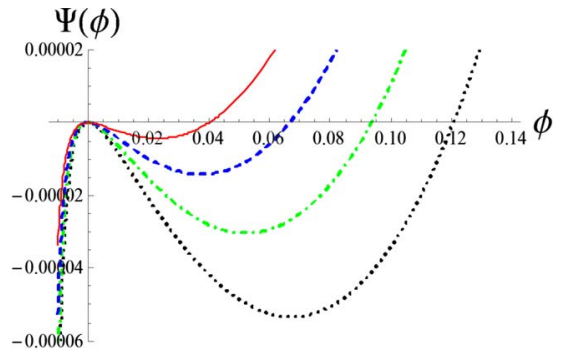

(a)

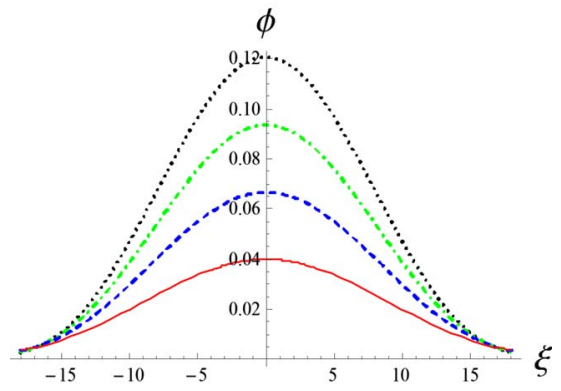

(b)

FIG. 10. (Color online) (a) Effect of obliqueness (via $\beta$ ) on the pseudopotential $\Psi(\phi)$ for $\kappa=15, M=0.95, \Omega_{i}=0.5$, and $\omega_{p i}=1.5$. From top to bottom: Solid curve: $\beta=0.97$; dashed curve: $\beta=0.96$; dot-dashed curve: $\beta=0.95$; dotted curve: $\beta=0.94$. (b) The corresponding electrostatic potential perturbations.

larger the angle $\theta$, the smaller the excitation should be, in order for the electrostatic approximation to be respected.

\section{Effect of magnetic field (via $\Omega_{i}$ )}

We have investigated the effect of the magnetic field on the solitary structure characteristics, as manifested via the appearance of the gyrofrequency $\Omega_{i}$. We find that the magnitude of the external magnetic field $B_{0}$ has no direct effect on the amplitude of the solitary waves (notice the fixed roots of $\Psi$ in Fig. 11). However, it does affect the width of the solitary waves. In fact, as $\Omega_{i}$ is increased, the depth of the pseudopotential well increases, i.e., a stronger magnetic field leads to steeper and thus narrower soliton profiles, as shown in Fig. 11. Finally, we note that the magnetic field has no effect on the velocity range, $\left[M_{1}, M_{2}\right]$, which is independent of $\Omega_{i}$, as we may see from Eq. (29).

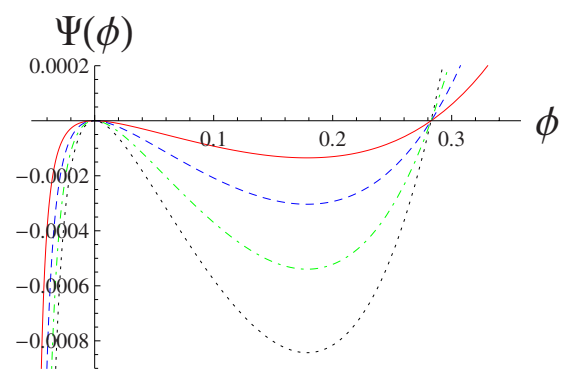

FIG. 11. (Color online) Magnetic field effect (via $\Omega_{i}$ ) for $\kappa=3, M=0.72$, $\beta=0.8, \omega_{p i}=1.3$. From top to bottom: Solid curve: $\Omega_{i}=0.2$; dashed curve: $\Omega_{i}=0.3$; dot-dashed curve: $\Omega_{i}=0.4$; dotted curve: $\Omega_{i}=0.5$. 


\section{CONCLUSIONS}

In this paper we have studied the nonlinear properties of arbitrary amplitude IA solitary waves propagating in a magnetized plasma of cold fluid ions and electrons with a kappa velocity distribution, i.e., for low values of $\kappa$, the electron distribution has an excess of superthermal particles compared with a Maxwellian distribution.

In the linear regime, we have obtained two dispersion curves, corresponding to the magnetized IA and ioncyclotron modes (modified on account of nonthermality and obliqueness of the propagation direction). We have shown that the frequency of the IA (ion-cyclotron) wave decreases (increases) with increasing obliqueness of propagation and the frequency of both waves decreases for an increased enhancement of superthermal electrons, i.e., lower values of $\kappa$.

It is found here that only positive potential IA solitary structures are observed in such a plasma, as is the case for a conventional electron-ion plasma in which the electrons are Maxwellian. Thus the excess superthermal electrons do not have a significant qualitative effect on the soliton behavior. In all soliton aspects, the typical characteristics associated with IA solitons in a plasma with a Maxwellian electron distribution ${ }^{26}$ are recovered for the limit, $\kappa \rightarrow \infty$.

The lower Mach number limit is $M_{1}=\cos \theta([2 \kappa-3] /$ $[2 \kappa-1])^{1 / 2}$, and it represents the true IA speed in the plasma model under discussion, i.e., $M_{1}$ is the speed of magnetized IA waves. Hence, as would be expected from standard theory, the solitons are indeed only found for superacoustic speeds, a point that appears to have been missed in the past. $^{34}$

On the other hand, although $M_{2}=[(2 \kappa-3) /(2 \kappa-1)]^{1 / 2}$ is the IA speed in an unmagnetized kappa plasma, it represents the upper limit for solitons, as it is the minimum value of the "upper" linear mode found here, viz., the cyclotron mode.

As is the case for Maxwellian electrons, ${ }^{34}$ the lower limit for the existence of IA solitons in a magnetized plasma with kappa distributed electrons is seen to be proportional to $\cos \theta$, where $\theta$ is the angle between the direction of propagation and the magnetic field. It may be added, for completeness that a recent study ${ }^{38}$ has, from first principles, drawn attention to the fact that great care needs to be exercised in the use of the pseudopotential method for oblique propagation. It was pointed out that only weakly oblique propagation should be considered if large amplitude excitations are to be modeled, in order that the electrostatic character of the excitations not be violated. ${ }^{38}$ Those general findings are to be respected and understood as the limits of our study.

We have shown that the lower soliton speed limit, $M_{1}$, decreases with an increase in excess superthermal component, i.e., with decreasing $\kappa$, and tends to zero as $\kappa \rightarrow 3 / 2$. The upper limit $\left(M_{2}\right)$ also decreases with decreasing $\kappa$, and, as $M_{1} / M_{2}=\cos \theta<1$, it follows that the range of accessible Mach numbers, $\left[M_{1}, M_{2}\right]$ decreases for decreasing $\kappa$, i.e., as the excess of superthermal particles is increased. These results are analogous to those for unmagnetized IA solitons. ${ }^{32}$ Further, the range $\left[M_{1}, M_{2}\right]$ also decreases with increasing propagation angle $\theta$ with respect to the magnetic field.

Both the amplitude and the profile steepness of solitons increase monotonically with the Mach number, for a given velocity distribution function, i.e., at fixed kappa, as was found for the unmagnetized case. ${ }^{32}$

We have also shown that for fixed Mach number, solitons in plasmas with a lower value of $\kappa$ have a larger amplitude and steeper profile than would be the case for solitons supported by a Maxwell-Boltzmann plasma. This is due to the lower soliton threshold speed $\left(M_{1}\right)$ that pertains in a low- $\kappa$ plasma containing increased excess superthermal ("tail") electrons. Hence $M-M_{1}$ increases as $\kappa$ is decreased, resulting in larger amplitude and steeper profile solitons. These results agree qualitatively with those found for an unmagnetized plasma. ${ }^{32}$

Finally, it is shown that the only effect of the magnetic field strength on the soliton behavior is that the soliton profile is steeper in a stronger magnetic field.

Our theoretical results on obliquely propagating IA waves in magnetized plasma can contribute to understanding localized electrostatic disturbances in space plasmas as well as in laboratory plasmas where a kappa distributed electron component is observed.

\section{ACKNOWLEDGMENTS}

This work was supported by a UK EPSRC Science and Innovation award to Queen's University Belfast Centre for Plasma Physics (Grant No. EP/D06337X/1). Enlightening discussions with Frank Verheest (Universiteit Gent, Belgium) are warmly acknowledged. N.S.S. would like to thank Guru Nanak Dev University, Amritsar, India for providing leave. The research is also supported in part by the National Research Foundation of South Africa (NRF). Any opinion, findings and conclusions or recommendations expressed in this material are those of the authors and therefore, NRF does not accept any liability in regard thereto.

\section{APPENDIX: DERIVATION OF EQS. (21)-(23)}

Substituting Eq. (18) into Eqs. (15) and (16)

$$
\begin{aligned}
& -\frac{M}{n} \frac{d u_{x}}{d \xi}+\alpha \frac{d \phi}{d \xi}-\frac{\Omega_{i}}{\omega_{p i}} u_{y}=0, \\
& -\frac{M}{n} \frac{d u_{y}}{d \xi}+\frac{\Omega_{i}}{\omega_{p i}} u_{x}=0 .
\end{aligned}
$$

Using the value of $u_{x}$ from Eq. (20), Eq. (A2) can be written as

$$
\frac{d u_{y}}{d \xi}=\frac{\Omega_{i}}{\omega_{p i}}\left[\frac{n}{\alpha}-\frac{1}{\alpha}-\frac{\beta^{2}}{M^{2} \alpha}\left(n \int n d \phi-n\right)\right] .
$$

Differentiating Eq. (A1) with respect to $\xi$ and substituting the value of $u_{x}$ and $d u_{y} / d \xi$, we get 


$$
\begin{aligned}
& \frac{3 M^{2}}{n^{4}}\left(\frac{d n}{d \xi}\right)^{2}-\frac{M^{2}}{n^{3}} \frac{d^{2} n}{d \xi^{2}}+\frac{d^{2} \phi}{d \xi^{2}} \\
& =\frac{\Omega_{i}^{2}}{\omega_{p i}^{2}}\left[n-1-\frac{\beta^{2} n}{M^{2}}\left(\int n d \phi-1\right)\right] \\
& \Rightarrow \frac{3 M^{2}}{n^{4}}\left(\frac{d n}{d \xi}\right)^{2}-\frac{M^{2}}{n^{3}} \frac{d^{2} n}{d \xi^{2}}+\frac{d^{2} \phi}{d \xi^{2}}=F(\phi) .
\end{aligned}
$$

Using Eq. (10), one writes $F(\phi)$ as a function of $\phi$

$$
\begin{aligned}
F(\phi)= & \frac{\Omega_{i}^{2}}{\omega_{p i}^{2}}\left[\left(1+\frac{\beta^{2}}{M^{2}}\right)\left(1-\frac{\phi}{\kappa-\frac{3}{2}}\right)^{-\kappa+1 / 2}\right. \\
& \left.-1-\frac{\beta^{2}}{M^{2}}\left(1-\frac{\phi}{\kappa-\frac{3}{2}}\right)^{-2 \kappa+2}\right] .
\end{aligned}
$$

Equation (A4) can be written in terms of electrostatic potential $\phi$ instead of normalized number density $n$ by using Eq. (10)

$$
\begin{aligned}
& \frac{M^{2}\left(\kappa-\frac{1}{2}\right)}{\left(\kappa-\frac{3}{2}\right)^{2}}(2 \kappa-2)\left(1-\frac{\phi}{\kappa-\frac{3}{2}}\right)^{2 \kappa-3}\left(\frac{d \phi}{d \xi}\right)^{2} \\
& \quad+\frac{M^{2}\left(\kappa-\frac{1}{2}\right)}{\left(\kappa-\frac{3}{2}\right)}\left(1-\frac{\phi}{\kappa-\frac{3}{2}}\right)^{2 \kappa-2} \frac{d^{2} \phi}{d \xi^{2}}+\frac{d^{2} \phi}{d \xi^{2}}=F(\phi) .
\end{aligned}
$$

Differentiating twice with respect to $\xi$, above equation reduces to

$$
\begin{aligned}
& \frac{M^{2}}{2} \frac{d^{2}}{d \xi^{2}}\left(1-\frac{\phi}{\kappa-\frac{3}{2}}\right)^{2 \kappa-1}+\frac{d^{2} \phi}{d \xi^{2}}=F(\phi) \\
& \Rightarrow \frac{d^{2}}{d \xi^{2}}\left[\phi+\frac{M^{2}}{2}\left(1-\frac{\phi}{\kappa-\frac{3}{2}}\right)^{2 \kappa-1}\right]=F(\phi) \Rightarrow \frac{d^{2} S}{d \xi^{2}}=F(\phi),
\end{aligned}
$$

where

$$
S=\phi+\frac{M^{2}}{2}\left(1-\frac{\phi}{\kappa-3 / 2}\right)^{2 \kappa-1} .
$$

Differentiating $S$ with respect to $\xi$ and squaring, we write

$$
\left(\frac{d S}{d \xi}\right)^{2}=[G(\phi)]^{2}\left(\frac{d \phi}{d \xi}\right)^{2},
$$

where $G(\phi)=1-M^{2}(\kappa-1 / 2)\left(1-\frac{\phi}{\kappa-3 / 2}\right)^{2 \kappa-2} /(\kappa-3 / 2)$. Multiplying both sides of Eq. (A6) by $d S / d \xi$, we can write

$$
\frac{d}{d \xi}\left[\frac{1}{2}\left(G(\phi) \frac{d \phi}{d \xi}\right)^{2}\right]=F(\phi) G(\phi) \frac{d \phi}{d \xi} .
$$

Integrating this equation under the boundary conditions $\phi \rightarrow 0$ and $d \phi / d \xi \rightarrow 0$ at $\xi \rightarrow \pm \infty$, one can obtain Eq. (23).

${ }^{1}$ V. M. Vasyliunas, J. Geophys. Res. 73, 2839, doi:10.1029/ JA073i009p02839 (1968).

${ }^{2}$ L. D. De Feiter and C. De Jager, Sol. Phys. 28, 183 (1973).

${ }^{3}$ J. D. Scudder, E. C. Sittler, and H. S. Bridge, J. Geophys. Res. 86, 8157, doi:10.1029/JA086iA10p08157 (1981).

${ }^{4}$ M. P. Leubner, J. Geophys. Res. 87, 6335, doi:10.1029/JA087iA08p06335 (1982).
${ }^{5}$ S. P. Christon, D. J. Williams, D. G. Mitchell, C. Y. Huang, and L. A. Frank, J. Geophys. Res. 96, 1, doi:10.1029/90JA01633 (1991).

${ }^{6}$ M. R. Collier, Geophys. Res. Lett. 20, 1531, doi:10.1029/93GL01702 (1993).

${ }^{7}$ D. T. Decker, B. Basu, J. R. Jasperse, D. J. Strikland, J. R. Sharber, and J. D. Winningham, J. Geophys. Res. 100, 21409, doi:10.1029/95JA01558 (1995).

${ }^{8}$ M. V. Codrescu, T. J. Fuller-Rowell, R. G. Robble, and D. S. Evans, J. Geophys. Res. 102, 19977, doi:10.1029/97JA01728 (1997).

${ }^{9}$ M. Maksimovic, S. P. Gary, and R. M. Skoug, J. Geophys. Res. 105, 18337, doi:10.1029/2000JA900039 (2000).

${ }^{10}$ E. E. Antonova, N. O. Ermakova, M. V. Stepanova, and M. V. Teltzov, Adv. Space Res. 31, 1229 (2003).

${ }^{11}$ H. Mori, M. Ishii, Y. Murayama, M. Kubota, K. Sakanoi, M. Y. Yamamoto, Y. Monzen, D. Lummerzheim, and B. J. Watkins, Ann. Geophys. 22, 1613 (2004)

${ }^{12}$ T. P. Armstrong, M. T. Paonessa, E. V. Bell, and S. M. Krimigis, J. Geophys. Res. 88, 8893, doi:10.1029/JA088iA11p08893 (1983).

${ }^{13}$ A. Hasegawa, K. Mima, and M. Duong-van, Phys. Rev. Lett. 54, 2608 (1985).

${ }^{14}$ M. A. Hellberg, R. L. Mace, R. J. Armstrong, and G. Karlstad, J. Plasma Phys. 64, 433 (2000).

${ }^{15}$ T. K. Baluku and M. A. Hellberg, Phys. Plasmas 15, 123705 (2008).

${ }^{16}$ M. A. Hellberg, R. L. Mace, T. K. Baluku, I. Kourakis, and N. S. Saini, Phys. Plasmas 16, 094701 (2009).

${ }^{17}$ W. C. Feldman, R. C. Anderson, J. R. Asbridge, S. J. Bame, J. T. Gosling, and R. D. Zwickl, J. Geophys. Res. 87, 632, doi:10.1029/ JA087iA02p00632 (1982).

${ }^{18}$ V. Pierrard and J. F. Lemaire, J. Geophys. Res. 101, 7923, doi:10.1029/ 95JA03802 (1996).

${ }^{19}$ M. Maksimovic, V. Pierrard, and J. F. Lemaire, Astron. Astrophys. 324, 725 (1997).

${ }^{20}$ S. Magni, H. E. Roman, R. Barni, C. Riccardi, Th. Pierre, and D. Guyomarc'h, Phys. Rev. E 72, 026403 (2005).

${ }^{21}$ R. A. Treumann, Astrophys. Space Sci. 277, 81 (2001).

${ }^{22}$ R. A. Treumann, C. H. Jaroschek, and M. Scholer, Phys. Plasmas 11, 1317 (2004).

${ }^{23}$ M. R. Collier, Adv. Space Res. 33, 2108 (2004).

${ }^{24}$ G. Livadiotis and D. J. McComas, J. Geophys. Res. 114, A11105, doi:10.1029/2009JA014352 (2009).

${ }^{25}$ C. Tsallis, J. Stat. Phys. 52, 479 (1988).

${ }^{26}$ R. Z. Sagdeev, in Reviews of Plasma Physics, edited by M. A. Leontovich (Consultants Bureau, New York, 1966), Vol. 4, pp. 23-91.

${ }^{27}$ F. F. Chen, Introduction to Plasma Physics and Controlled Fusion (Plenum, New York, 1984).

${ }^{28}$ R. Bharuthram and P. K. Shukla, Planet. Space Sci. 40, 973 (1992).

${ }^{29}$ F. Verheest, M. A. Hellberg, and G. S. Lakhina, Astrophys. Space Sci. Trans. 3, 15 (2007).

${ }^{30}$ F. Verheest, in Aspects of Plasma Physics, edited by P. K. Shukla, B. Eliasson, L. Stenflo, and R. Bingham (World Scientific, Singapore, 2008), p. 316.

${ }^{31}$ F. Verheest, M. A. Hellberg, and I. Kourakis, Phys. Plasmas 15, 112309 (2008).

${ }^{32}$ N. S. Saini, I. Kourakis, and M. A. Hellberg, Phys. Plasmas 16, 062903 (2009).

${ }^{33}$ M. Y. Yu, P. K. Shukla, and S. Bujarbarua, Phys. Fluids 23, 2146 (1980).

${ }^{34}$ L. C. Lee and J. R. Kan, Phys. Fluids 24, 431 (1981).

${ }^{35}$ M. K. Kalita and S. Bujarbarua, Plasma Phys. 25, 1369 (1983).

${ }^{36}$ Yashvir, T. N. Bhatnagar, and S. R. Sharma, Plasma Phys. Controlled Fusion 26, 1303 (1984).

${ }^{37}$ S. Mahmood, A. Mushtaq, and H. Saleem, New J. Phys. 5, 28 (2003).

${ }^{38}$ F. Verheest, J. Phys. A: Math. Theor 42, 285501 (2009).

${ }^{39}$ R. L. Mace and M. A. Hellberg, Phys. Plasmas 2, 2098 (1995).

${ }^{40}$ M. A. Hellberg and R. L. Mace, Phys. Plasmas 9, 1495 (2002).

${ }^{41}$ R. L. Mace and M. A. Hellberg, Phys. Plasmas 16, 072113 (2009).

${ }^{42}$ See, e.g., pp. $105-109$ in Ref. 27.

${ }^{43}$ Y. F. Chateau and N. Meyer-Vernet, J. Geophys. Res. 96, 5825, doi:10.1029/90JA02565 (1991).

${ }^{44}$ D. A. Bryant, J. Plasma Phys. 56, 87 (1996).

${ }^{45}$ R. L. Mace, M. A. Hellberg, and R. A. Treumann, J. Plasma Phys. 59, 393 (1998).

${ }^{46}$ R. L. Mace, G. Amery, and M. A. Hellberg, Phys. Plasmas 6, 44 (1999).

${ }^{47}$ M.-J. Lee, Phys. Plasmas 14, 032112 (2007). 